\title{
Is reduced Planck's constant- an outcome of electroweak gravity?
}

\author{
U.V.S. Seshavatharam ${ }^{1}$ and S. Lakshminarayana ${ }^{2}$ \\ ${ }^{1}$ Honorary faculty, I-SERVE, Survey no-42, Hitech city, Hyderabad-84,Telangana, INDIA \\ ${ }^{2}$ Dept. of Nuclear Physics, Andhra University, Visakhapatnam-03,AP, INDIA \\ Emails: seshavatharam.uvs@gmail.com (and) lnsrirama@gmail.com \\ Orcid numbers : 0000-0002-1695-6037 (and) 0000-0002-8923-772X
}

\begin{abstract}
To understand the mystery of final unification, in our earlier publications, we proposed that there exist three atomic gravitational constants associated with electroweak, strong and electromagnetic interactions. During cosmic evolution, if one is willing to give equal importance to Higgs boson and Planck mass in understanding the massive origin of elementary particles, then it seems quite logical to expect a common relation in between Planck scale and Electroweak scale. Based on these two points, we noticed that, electroweak field seems to be operated by a primordial massive fermion of rest energy $585 \mathrm{GeV}$. It can be considered as the zygote of all elementary particles and galactic dark matter. H-bar seems to be a characteristic outcome of unified electroweak gravity. Electron rest mass seems to be a characteristic outcome of electroweak and strong gravity. Proton rest mass seems to be a characteristic outcome of electroweak, strong and electromagnetic gravity. Recently observed $3.5 \mathrm{keV}$ photon seems to be an outcome of annihilation of charged baby lepton of rest energy $1.75 \mathrm{keV}$. Interesting point to be noted is that, Schwarzschild radius of electron is 0.48 nanometer and it needs further investigation with respect to emerging nanoscience and technology. Proceeding further, by considering electromagnetic and weak gravitational constants, neutron life time can be understood.
\end{abstract}

Key words: Four gravitational constants; Electroweak fermion; Reduced Planck's constant; Stellar mass limits; $3.5 \mathrm{keV}$ photon; Neutron life time;

\begin{tabular}{|c|c|c|c|}
\hline \multicolumn{4}{|c|}{ Nomenclature } \\
\hline & Newtonian gravitational constant $=G_{N}$ & 18) & Nuclear charge radius $=R_{(Z, A)}$ \\
\hline & Electromagnetic gravitational constant $=G_{e}$ & 19) & Schwarzschild radius of $M_{w}=R_{w}$ \\
\hline 3) & Nuclear gravitational constant $=G_{s}$ & 20) & Schwarzschild radius of $m_{e}=R_{e}$ \\
\hline 4) & Weak gravitational constant $=G_{W}$ & 21) & Schwarzschild radius of atom $=R_{\text {atom }}$ \\
\hline 5) & Fermi's weak coupling constant $=G_{F}$ & 22) & Mean stable mass number $=A_{m}$ \\
\hline 6) & Electroweak fermion $=M_{w}$ & 23) & Nuclear binding energy $=B_{(A, Z)}^{m}$ \\
\hline & $\begin{array}{l}\text { Reduced Planck's constant }=\hbar \\
\text { Speed of light }=c\end{array}$ & 24) & Nuclear binding energy coefficient $=$ \\
\hline & Strong coupling constant $=\alpha_{s}$ & 25) & $\begin{array}{l}D_{0} \\
\text { Coefficients connected with nuclear }\end{array}$ \\
\hline & Elementary charge $=e$ & & stability and binding energy $=\left(k_{1}, k_{2}\right)$ \\
\hline & Mass of proton $=m_{p}$ & 26) & Mass limit of stellar object $=M_{X}$ \\
\hline & Mass of neutron $=m_{n}$ & 27) & Characteristic ratio associated with \\
\hline & Mass of electron $=m_{e}$ & & $\longdiv { 4 \pi \varepsilon _ { 0 } G _ { \rho } m _ { \rho } ^ { 2 } }$ \\
\hline 14) & Mass of Up quark $=m_{u}$ & & 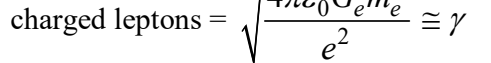 \\
\hline & Mass of Down quark $=m_{d}$ & & Mass of charged haby lenton $=(m)^{ \pm}$ \\
\hline & Bohr Radius $=a_{0}$ & 28) & $=\left(m_{x l}\right)$ \\
\hline & Schwarzschild radius of $m_{p}=R_{p}$ & 29) & Neutron life time $=t_{n}$ \\
\hline
\end{tabular}

\section{Introduction}

Even though celestial objects that show gravity are confirmed to be made up of so many atoms, so far scientists could not find any relation in between gravity and the atomic interactions at quantum gravity level $[1,2]$. Black hole temperature point of view [3], strong interaction point of view [4-7] and 
electroweak interaction point of view [8], scientists found very interesting similarities in between gravity and quantum phenomena. Quantum cosmology point of view [9] and nuclear quantum gravity point of view [10-20], authors could develop workable ideas, concepts and relations. On a whole, workability is still lagging. It clearly indicates that, there is something wrong in our notion of understanding or there is something missing in developing the unified physical concepts and needs a critical review at fundamental level. In this context, we hope that, electroweak scale $[21,22,23]$ can certainly yield useful stuff.

\section{Motivating concepts}

To develop new and workable ideas, we wish to highlight the following points.

1) During cosmic evolution, if one is willing to give equal importance to Higgs boson and Planck mass in understanding the massive origin of elementary particles and observed matter [24,25], then it seems quite logical to expect a common relation in between Planck scale and Electroweak scale.

2) Whether particle's massive nature is due to electromagnetism or gravity or weak interaction or strong interaction or cosmic dust or dark matter [26] or something else, is unclear.

3) Without understanding the massive nature, it is not reasonable to classify the field created by any elementary particle.

4) All the four interactions seem to be associated with $(\hbar)$.

5) Nobody knows the mystery of $(\hbar)$ which seems to be a basic measure of angular momentum [27,28,29,30].

6) Nobody knows the mystery of existence, stability and behavior of 'proton' or 'electron'.

7) 'Mass' is a basic property of space-time curvature and basic ingredient of angular momentum.

8) Atoms are mainly characterized by protons and electrons.

9) 'Free neutron' is an unstable particle.

\section{Basic assumptions}

1) There exists a characteristic electroweak fermion of rest energy [18], $M_{w} c^{2} \cong 584.725 \mathrm{GeV}$.
2) $M_{w}$ can be considered as the zygote of all elementary particles.

3) Fermi's weak coupling constant $\left(G_{F}\right)[29,30,31]$ can be considered as the basic unified coupling constant.

4) Each atomic interaction is associated with a characteristic gravitational coupling constant.

$$
\begin{aligned}
& G_{e} \cong 2.374335 \times 10^{37} \mathrm{~m}^{3} \mathrm{~kg}^{-1} \mathrm{sec}^{-2} \\
& G_{s} \cong 3.329561 \times 10^{28} \mathrm{~m}^{3} \mathrm{~kg}^{-1} \mathrm{sec}^{-2} \\
& G_{w} \cong 2.909745 \times 10^{22} \mathrm{~m}^{3} \mathrm{~kg}^{-1} \mathrm{sec}^{-2} \\
& G_{N} \cong 6.679855 \times 10^{-11} \mathrm{~m}^{3} \mathrm{~kg}^{-1} \mathrm{sec}^{-2}
\end{aligned}
$$

\section{Characteristic unified relations}

Based on the above points, we propose the following new and workable relations.

$$
\begin{aligned}
& \hbar c \cong G_{w} M_{w}^{2} \cong \sqrt{G_{F}\left(\frac{c^{4}}{4 G_{w}}\right)} \\
& \Rightarrow \hbar \cong \frac{G_{w} M_{w}^{2}}{c} \cong \sqrt{\frac{G_{F} c^{2}}{4 G_{w}}}
\end{aligned}
$$

where $\left(\frac{c^{4}}{4 G_{w}}\right) \cong 6.9401 \times 10^{10} \mathrm{~N}$ is the characteristic force associated with electroweak interaction.

$$
\begin{gathered}
m_{e} \cong\left(\frac{G_{w}}{G_{s}}\right) M_{w} \\
m_{p} \cong\left(\frac{G_{s}}{G_{w}}\right)\left(\frac{G_{s}}{G_{e}}\right) M_{w} \cong\left(\frac{G_{s}^{2}}{G_{w} G_{e}}\right) M_{w} \\
\frac{m_{p}}{m_{e}} \cong \frac{G_{s}^{3}}{G_{w}^{2} G_{e}}
\end{gathered}
$$

\section{Specific unified relations connected with $G_{N}$}

With reference to Newtonian gravitational constant [32-39],

$$
\left(\frac{m_{p}}{m_{e}}\right)^{10} \cong\left(\frac{G_{w}}{G_{N}}\right)
$$




$$
\exp \left(\frac{1}{\alpha_{s}^{2}}\right) \cong\left(\frac{G_{w}}{G_{N}}\right)
$$

where $\alpha_{\mathrm{s}}=$ Strong coupling constant $[29,31]$

$$
\frac{m_{p}}{m_{e}} \cong\left(\frac{G_{s}}{G_{e}^{1 / 3} G_{N}^{2 / 3}}\right)^{1 / 7}
$$

\section{Specific unified relations connected with nuclear radius and Bohr radius}

Characteristic Schwarzschild radius of proton and Schwarzschild radius of atom can be addressed with the following relations.

$R_{p} \cong \frac{2 G_{s} m_{p}}{c^{2}} \cong 1.2393 \mathrm{fm}$

$=$ Characteristic nuclear charge radius $[40,41]$

$$
R_{(Z, A)} \cong\left\{Z^{1 / 3}+(\sqrt{Z(A-Z)})^{1 / 3}\right\}\left(\frac{G_{s} m_{p}}{c^{2}}\right)
$$

$=$ Nuclear charge radius [42]

$$
\begin{aligned}
a_{0} & \cong\left(\frac{4 \pi \varepsilon_{0} G_{e} m_{e}^{2}}{e^{2}}\right)\left(\frac{G_{s} m_{p}}{c^{2}}\right) \cong 5.2918 \times 10^{-11} \mathrm{~m} \\
& =\text { Bohr radius of Hydrogen atom [28] }
\end{aligned}
$$

\section{Specific unified relations connected with proton-electron mass ratio}

With reference to electroweak interaction,

$$
\begin{aligned}
R_{w} \cong \frac{2 G_{w} M_{w}}{c^{2}} \cong 6.7494 \times 10^{-19} \mathrm{~m} \\
=\text { Schwarzschild radius of } M_{w} \\
\frac{R_{p}}{R_{w}} \cong\left(\frac{2 G_{s} m_{p}}{c^{2}}\right) \div\left(\frac{2 G_{w} M_{w}}{c^{2}}\right) \cong \frac{G_{s} m_{p}}{G_{w} M_{w}} \cong\left(\frac{m_{p}}{m_{e}}\right)
\end{aligned}
$$

With reference to $R_{w} \cong 6.7494 \times 10^{-19} \mathrm{~m}$ and cconsidering $\left(\frac{m_{p}}{m_{e}}\right)$ as a geometric ratio, nuclear radius and atomic radius can be estimated in the following way.

$$
R_{1} \cong\left(\frac{m_{p}}{m_{e}}\right)\left(\frac{2 G_{w} M_{w}}{c^{2}}\right) \cong 1.2393 \mathrm{fm}
$$

$$
R_{2} \cong\left(\frac{m_{p}}{m_{e}}\right)^{2}\left(\frac{2 G_{w} M_{w}}{c^{2}}\right) \cong 2.275 \mathrm{pm}
$$

With reference to electromagnetic gravitational constant, Schwarzschild radius of electron can be addressed with,

$$
R_{e} \cong\left(\frac{2 G_{e} m_{e}}{c^{2}}\right) \cong 0.48 \mathrm{~nm}
$$

Based on relations (14) and (15), identifying $R_{2}$ and $R_{e}$ as characteristic length scales associated with characteristic atomic radius, we noticed that,

$$
\begin{aligned}
& \sqrt{R_{2} R_{e}} \cong\left(\frac{2 \sqrt{G_{e} G_{s}} m_{p}}{c^{2}}\right) \cong 33.1 \mathrm{pm} \\
& \cong R_{\text {atom }} \cong \text { Scwarzschild radius of atom [43] }
\end{aligned}
$$

\section{Specific unified relations connected with nuclear stability and binding energy}

Nuclear mean stability and binding energy $[44,45]$ can be understood with the following two relations.

Nuclear mean stability can be understood with,

$$
\begin{aligned}
& \left(A_{s}\right)_{\text {mean }} \cong A_{m} \cong 2 Z+k_{1} Z^{2} \\
& \text { where }\left\{\begin{array}{l}
k_{1} \cong 4\left(\frac{G_{s}^{2}}{G_{e} G_{w}}\right) \cong 4\left(\frac{m_{p}}{M_{w}}\right) \\
\cong 0.0064185
\end{array}\right\}
\end{aligned}
$$

Nuclear binding energy can be understood with,

$$
B_{(A, Z)} \cong\left\{\begin{array}{l}
\left(1-k_{2} \sqrt{Z N}\right) A-A^{1 / 3} \\
-\left(1+\frac{\left(A_{m}-A\right)^{2}}{A_{m}}\right)
\end{array}\right\}\left(B_{0} \cong 10.1 \mathrm{MeV}\right)
$$

where, $k_{2} \cong 2 \sqrt{\frac{G_{w}}{G_{s}}} \cong 2 \sqrt{\frac{m_{e}}{M_{w}}} \cong 0.00189$

$B_{0} \cong\left(\frac{1}{\alpha_{s}}\right) \frac{e^{2}}{4 \pi \varepsilon_{0} R_{0}}$

$\frac{\cong\left[\left(2 m_{u}+m_{d}\right) c^{2}+\left(m_{u}+2 m_{d}\right) c^{2}\right]}{2} \cong 10.275 \mathrm{MeV}$

where $\left(m_{u}, m_{d}\right)=\mathrm{Up}$ and Down quark masses [29] 
Note-: The numbers $\left(k_{1}\right.$ and $\left.k_{2}\right)$ can be considered as the characteristic outcomes of the combined effect of strong and electromagnetic coupling constants. With trial-error method, we noticed that, $\left(k_{1}, k_{2}\right) \cong\left(\frac{\left(1-\alpha_{s}\right)^{n}}{2 n-1}\right) \alpha \cong(0.00644,0.001892)$ where $n \cong 1,2$ and $\alpha_{s} \cong 0.1181$. It needs further study.

\section{Specific unified relations connected with stellar mass limits}

With reference to strong nuclear gravitational constant and astro-physics point of view $[14,16]$, by considering nucleon as a characteristic building block, stellar mass limit $[46,47]$ can be understood with a relation of the form,

$$
\frac{G_{N} M_{X}}{G_{s} m_{n}} \cong \sqrt{\frac{G_{s}}{G_{N}}}
$$

Thus, characteristic stellar mass limit can be estimated with a very simple relation of the form,

$$
M_{X} \cong\left(\frac{G_{s}}{G_{N}}\right)^{\frac{3}{2}}\left(m_{n}\right) \cong 9.37 \text { solar masses }
$$

Another interesting relation is,

$$
\begin{gathered}
\frac{G_{N} M_{X}}{G_{s} \sqrt{m_{n} M_{w}}} \cong \sqrt{\frac{G_{s}}{G_{N}}} \\
M_{X} \cong\left(\frac{G_{s}}{G_{N}}\right)^{\frac{3}{2}} \sqrt{m_{n} M_{w}} \cong 234 \text { solar masses }
\end{gathered}
$$

With reference to electromagnetic gravitational constant, mass limits of super massive stellar objects can be understood.

\section{Applications of $G_{e}$ in elementary particle physics and astrophysics}

\section{A) Understanding the recently observed 3.5 keV galactic photon}

Recent galactic X-ray [48,49] studies strongly confirm the existence of a new photon of energy 3.5 $\mathrm{keV}$. So far, its origin is unknown and unclear. In this context, we propose the following alternative mechanism for understanding the origin of $3.5 \mathrm{keV}$ photon.

1) There exists a characteristic charged baby lepton of rest mass,

$$
\left(m_{x l}\right)^{ \pm} \cong \sqrt{\frac{e^{2}}{4 \pi \varepsilon_{0} G_{e}}} \cong 1.75 \mathrm{keV} / c^{2}
$$

2) With pair annihilation mechanism, $\left(m_{x l}\right)$ generates a photon of rest energy $3.5 \mathrm{keV}$

3) With current and future particle accelerators, $\left(m_{x l}\right)^{ \pm} \cong 1.75 \mathrm{keV} / c^{2}$ can be generated.

\section{B) Fitting Muon and Tau rest masses}

Experimentally observed [29] Muon and Tau rest masses can be fitted in the following way.

$$
\left.m_{(\mu, \tau)} c^{2} \cong\left[\gamma^{3}+\left(n^{2} \gamma\right)^{n}\left(\frac{G_{e}}{G_{N}}\right)^{1 / 4}\right]^{\frac{1}{3}} 1.75 \mathrm{keV}\right\}
$$

where,

$\gamma \cong \sqrt{\frac{4 \pi \varepsilon_{0} G_{e} m_{e}^{2}}{e^{2}}} \cong 292.187$ and $n=1$ and 2

For $n=1$, obtained $m_{\mu} c^{2} \cong 106.5 \mathrm{MeV}$

$n=2$, obtained $m_{\tau} c^{2} \cong 1781.5 \mathrm{MeV}$.

At $n=3$, a new heavy charged lepton of rest energy $42.2 \mathrm{GeV}$ can be predicted.

11. Specific unified relations connected with

$$
\left(G_{e}, G_{s}, G_{w}, G_{N}\right)
$$

Based on the above relations and applications, we propose the following three relations.

$$
\begin{gathered}
\frac{M_{w}}{m_{e}} \cong \frac{G_{w}^{5 / 2} G_{e}^{5 / 3}}{G_{s}^{4} G_{N}^{1 / 6}} \\
\frac{M_{w}}{m_{p}} \cong \frac{G_{s}^{1 / 2} G_{e}^{1 / 6} G_{N}^{1 / 12}}{G_{w}^{3 / 4}} \\
\frac{m_{p}}{m_{e}} \cong \frac{G_{w}^{13 / 4} G_{e}^{3 / 2}}{G_{s}^{9 / 2} G_{N}^{1 / 4}}
\end{gathered}
$$

Based on these relations,

$$
G_{N} \cong\left(\frac{m_{e}}{m_{p}}\right)^{4} \frac{G_{w}^{13} G_{e}^{6}}{G_{s}^{18}}
$$


Based on the nuclear experiments and astrophysical observations,

1) $\left(G_{e}\right)$ can be estimated from relation (23).

2) $\left(G_{s}\right)$ can be estimated from relations (8-10).

3) $\left(G_{w}\right)$ can be estimated from relation (1).

\section{Understanding neutron life time with $\left(G_{e}, G_{w}\right)$}

One of the key objectives of any unified description is to simplify or eliminate the complicated issues of known physics. Neutron life estimation is one of such complicated issue $[29,50,51]$. In this context, in our earlier publications [20,52], we proposed the following relations.

$$
\left.\begin{array}{l}
t_{n} \cong\left(\frac{G_{e}}{G_{w}}\right)\left(\frac{G_{e} m_{n}^{2}}{\left(m_{n}-m_{p}\right) c^{3}}\right) \\
\cong\left(\frac{G_{e}^{2} m_{n}^{2}}{G_{w}\left(m_{n}-m_{p}\right) c^{3}}\right) \cong 874.94 \mathrm{sec}
\end{array}\right\}
$$

Plausible point to be noted is that, relativistic mass of neutron seems to play a crucial role in understanding the increasing neutron life time. It can be understood with,

$$
t_{n} \propto \frac{m_{n}^{2}}{\left[1-\left(v^{2} / c^{2}\right)\right]} \text { and } t_{n} \cong \frac{874.94 \mathrm{sec}}{\left[1-\left(v^{2} / c^{2}\right)\right]}
$$

In this way, bottle method [50] and beam method [51] of neutron life time experiments can be correlated with confined and moving neutrons.

\section{Discussion}

We appeal that,

(1) Success of any unified model depends on its ability to involve gravity in microscopic models.

(2) Full-fledged implementation of gravity in microscopic physics must be able to:
a) Estimate the ground state elementary particle rest masses of the three atomic interactions.
b) Estimate the coupling constants of the three atomic interactions.
c) Estimate the range of all interactions.
d) Estimate the Newtonian gravitational constant.

(3) As the root/path is unclear and unknown, to make it success or to have a full-fledged implementation, one may be forced to consider a new path that may be out-of-scope of the currently believed string theory models[53].

(4) In our approach,

a) We assign a different gravitational constant for each basic interaction.

b) Considering $585 \mathrm{GeV}$ fermion as the characteristic building block of all elementary particles, an attempt is made to fit proton and electron masses.

c) During this journey, without considering arbitrary numbers or coefficients, we come across many strange and interesting relations for estimating other atomic and nuclear coupling constants.

d) Based on relations (5) and (6), magnitudes of $\left(G_{w}, \alpha_{s}\right)$ can be estimated in a verifiable approach.

\section{Conclusion}

With further study, research and confirming the existence of $M_{w} c^{2} \cong 584.725 \mathrm{GeV}$, actual essence of final unification can be understood.

\section{Acknowledgements}

Author Seshavatharam is indebted to professors shri M. Nagaphani Sarma, Chairman, shri K.V. Krishna Murthy, founder Chairman, Institute of Scientific Research in Vedas (I-SERVE), Hyderabad, India and Shri K.V.R.S. Murthy, former scientist IICT (CSIR), Govt. of India, Director, Research and Development, I-SERVE, for their valuable guidance and great support in developing this subject.

\section{References}

[1] Frank Wilczek. QCD made simple (PDF). Physics Today. 53 (8): 22-28 (2000)

[2] M. Bojowald. Quantum cosmology: a review. Rep. Prog. Phys. 78 (2015) 023901

[3] Hawking, S.W. Particle Creation by Black Holes. Communications in Mathematical Physics, 43, 199-220(1975)

[4] K. Tennakone, Electron, muon, proton, and strong gravity. Phys. Rev. D, 10, 1722 (1974)

[5] C. Sivaram and K. Sinha, Strong gravity, black holes, and hadrons. Physical Review D., 16(6), 1975-1978 (1977) 
[6] De Sabbata V and M. Gasperini. Strong gravity and weak interactions. Gen. Relat. Gravit. 10, 9, 731-741, (1979)

[7] Salam A, Sivaram C. Strong Gravity Approach to QCD and Confinement. Mod. Phys. Lett., v. A8(4), 321-326 (1993)

[8] Roberto Onofrio. On weak interactions as shortdistance manifestations of gravity. Modern Physics Letters A 28, 1350022 (2013)

[9] Seshavatharam UVS and Lakshminarayana S. A Practical Model of Godel-Planck- Hubble-Birch Universe. Athens Journal of Sciences- Vol.6, Issue 3, 211-230 (2019)

[10] Seshavatharam UVS, Lakshminarayana S. To confirm the existence of atomic gravitational constant. Hadronic Journal.34(4):379 (2011)

[11] Seshavatharam UVS, Lakshminarayana S. Molar electron mass and the basics of TOE. Journal of Nuclear and Particle Physics. 2(6):132 -141 (2012)

[12] Seshavatharam UVS et al. Understanding the constructional features of materialistic atoms in the light of strong nuclear gravitational coupling. Materials Today: 3/10PB, Proceedings 3, 39763981 (2016)

[13] Seshavatharam UVS and Lakshminarayana S. Towards a workable model of final unification. International Journal of Mathematics and Physics 7(1), 117-130 (2016)

[14] Seshavatharam UVS and Lakshminarayana S. Understanding the basics of final unification with three gravitational constants associated with nuclear, electromagnetic and gravitational interactions. Journal of Nuclear Physics, Material Sciences, Radiation and Applications 4(1),1-19 (2017)

[15] Seshavatharam UVS and Lakshminarayana S. On the role of 'reciprocal' of the strong coupling constant in nuclear structure. Journal of Nuclear Sciences, 4( 2), 31-44 (2017).

[16] Seshavatharam UVS and Lakshminarayana S. Applications of gravitational model of possible final unification in both large and small scale physics. Prespacetime journal, Vol 7, issue2, 405-421 (2016)

[17] Seshavatharam UVS and Lakshminarayana S. A virtual model of microscopic quantum gravity, Prespacetime Journal, 9(1), 58-82 (2018)

[18] Seshavatharam UVS and Lakshminarayana S. On the role of four gravitational constants in nuclear structure. Mapana Journal of Sciences, 18(1), 2145 (2019)

[19] Seshavatharam UVS and Lakshminarayana S. On the Role of Large Nuclear Gravity in Understanding Strong Coupling Constant, Nuclear Stability Range, Binding Energy of Isotopes and Magic proton numbers - A Critical
Review. J. Nucl. Phys. Mat. Sci. Rad. A. 6(2), 142-160 (2019)

[20] Seshavatharam UVS and Lakshminarayana S. On the Role of Squared Neutron Number in Reducing Nuclear Binding Energy in the Light of Electromagnetic, Weak and Nuclear Gravitational Constants - A Review. Asian Journal of Research and Reviews in Physics, 2(3): 1-22, (2019)

[21]F. Englert and R. Brout. Broken Symmetry and the Mass of Gauge Vector Mesons. Physical Review Letters, vol. 13, Issue 9, pp. 321-323 (1964)

[22] Higgs P. Broken Symmetries and the Masses of Gauge Bosons. Physical Review Letters. 13 (16): 508-509 (1964)

[23] The ATLAS Collaboration. Observation of a new particle in the search for the Standard Model Higgs boson with the ATLAS detector at the LHC. Phys.Lett. B716, 1-29 (2012)

[24]Ralph A. Alpher, George Gamow and Robert Herman.Thermal cosmic radiation and the formation of protogalaxies. PNAS, 58 (6) 2179 2186. (1967)

[25]G. Gamow. The Evolution of the Universe. Nature 162, 680-682 (1948)

[26] Berezhiani L, Khoury J and Wang J. Universe without Dark Energy: Cosmic Acceleration from Dark Matter-baryon Interactions. Physical Review 95(12-15): 123530 (2017)

[27] Max Planck. Ueber das Gesetz der Energieverteilung im Normalspectrum (PDF). Ann. Phys., 309 (3): 553-63 (1901)

[28] Niels Bohr. On the Constitution of Atoms and Molecules, Part I (PDF). Philosophical Magazine. 26 (151): 1-24 Niels Bohr (1913)

[29] M. Tanabashi et al. (Particle Data Group), Phys. Rev. D 98, 030001 (2018)

[30]Fermi E. Tentativo di una teoria dei raggi $\beta$. La Ricerca Scientifica (in Italian). 2 (12), (1933).

[31]P. J. Mohr, D. B. Newell, and B. N. Taylor, CODATA recommended values of the fundamental constants: Rev. Mod. Phys. 88, 035009 (2014)

[32] B. Canuel et al. Exploring gravity with the MIGA large scale atom interferometer. Science reports, 8:14064 (2018)

[33] Christos Merkatas et al. Shades of dark uncertainty and consensus value for the Newtonian constant of gravitation. arXiv:1905.09551v1 (2019)

[34] Li, Qing et al. Measurements of the gravitational constant using two independent methods. Nature 560, 582-588 (2018)

[35]G. Rosi, F. Sorrentino, L. Cacciapuoti, M. Prevedelli, and G. M. Tino. Precision measurement of the Newtonian gravitational 
constant using cold atoms, Nature 510 (7506), 518-521 (2014)

[36] S. Schlamminger and R.D. Newman. Recent measurements of the gravitational constant as a function of time. Phys. Rev. D 91, 121101 (2015)

[37]C. Rothleitner and S. Schlamminger. Measurements of the Newtonian constant of gravitation, G. Rev. Sci. Instrum. 88, 111101 (2017)

[38] G. Rosi, Challenging the big $G$ measurement with atoms and light. J. Phys. B: At., Mol. Opt. Phys. 49(20), 202002 (2016)

[39] Seshavatharam UVS and Lakshminarayana S. On the role of Newtonian gravitational constant in estimating Proton-Electron mass ratio and baryon mass spectrum. International Journal of Innovative Studies in Sciences Engineering Technology. Vol 5, Issue 10, 18-21 (2019)

[40]Rutherford E. The scattering of $\alpha$ and $\beta$ particles by matter and the structure of the atom. The London, Edinburgh, and Dublin Philosophical Magazine and Journal of Science. Series 6. 21 (125): (1911)

[41]Hofstadter R. et al. High-Energy Electron Scattering and Nuclear Structure Determinations. Phys. Rev. 92, 978 (1953)

[42] T. Bayram, S. Akkoyun, S. O. Kara and A. Sinan, New Parameters for Nuclear Charge Radius Formulas, Acta Physica Polonica B. 44( 8), 17911799 (2013)

[43] https://en.wikipedia.org/wiki/Atomic_radii_of_th e_elements_(data_page)
[44] Seshavatharam UVS and Lakshminarayana S. Implications and Applications of Fermi Scale Quantum Gravity. Preprints 2019, 2019110134.

[45] Seshavatharam UVS and Lakshminarayana S. Hypothetical Role of Large Nuclear Gravity in Understanding the Significance and Applications of the Strong Coupling Constant in the Light of Up and Down Quark Clusters. Preprints 2019, 2019110398

[46] Astrophysics and Space Science Library. 412, Very Massive Stars in the Local Universe. Editor Jorick S. Vink. Springer (2014)

[47] https://openstax.org/books/astronomy/pages/18-2measuring-stellar-masses

[48] Eric Carlson et al. Where do the $3.5 \mathrm{keV}$ photons come from? A morphological study of the Galactic Center and of Perseus. JCAP 02, 009 (2015)

[49] S Riemer-Sorensen. Questioning a $3.5 \mathrm{keV}$ dark matter emission line. A\&A 590, A71 (2016)

[50] RW Pattie Jr et al. Measurement of the neutron lifetime using a magneto-gravitational trap and in situ detection. Science 11, Vol. 360, Issue 6389, pp. 627-632 (2018)

[51]F. E. Wietfeldt et al. A path to a $0.1 \mathrm{~s}$ neutron lifetime measurement using the beam method. Physics Procedia 51, 54 - 58 ( 2014 )

[52] Seshavatharam UVS and Lakshminarayana S. Neutron life time enigma in the light of Electromagnetic and Weak gravitational constants. Nucleus-2019, Dubna, Russia, Book of abstracts, Page 242 (2019)

[53] Joseph Polchinski. String theory to the rescue. arXiv:1512.02477v5 [hep-th] (2015) 